University of Nebraska - Lincoln

DigitalCommons@University of Nebraska - Lincoln

August 1978

\title{
Effect of random anisotropy on magnetic properties of amorphous systems
}

\author{
J.D. Patterson \\ University of Nebraska - Lincoln \\ G.R. Gruzalski \\ University of Nebraska - Lincoln \\ David J. Sellmyer \\ University of Nebraska-Lincoln, dsellmyer@unl.edu
}

Follow this and additional works at: https://digitalcommons.unl.edu/physicssellmyer

Part of the Physics Commons

Patterson, J.D.; Gruzalski, G.R.; and Sellmyer, David J., "Effect of random anisotropy on magnetic properties of amorphous systems" (1978). David Sellmyer Publications. 172.

https://digitalcommons.unl.edu/physicssellmyer/172

This Article is brought to you for free and open access by the Research Papers in Physics and Astronomy at DigitalCommons@University of Nebraska - Lincoln. It has been accepted for inclusion in David Sellmyer Publications by an authorized administrator of DigitalCommons@University of Nebraska - Lincoln. 


\title{
Effect of random anisotropy on magnetic properties of amorphous systems
}

\author{
J. D. Patterson, ${ }^{*}$ G. R. Gruzalski, ${ }^{\dagger}$ and D. J. Sellmyer \\ Behlen Laboratory of Physics, The University of Nebraska, Lincoln, Nebraska 68588
}

(Received 16 January 1978)

\begin{abstract}
We consider the random anisotropy model for amorphous magnetism by making a local-mean-field approximation (LMFA) on arrays of spin-one particles. Hysteresis loops and the temperature $(T)$ dependence of several thermodynamic quantities are presented for various values of the ratio of the strength of the exchange $(J)$ to the strength of the uniaxial anisotropy $(D)$. Using the LMFA limits us to systems with a small number $(N)$ of spins, of which we explicitly consider $N=64,216$, and 1000 . We assume periodic boundary conditions on a system with $N^{1 / 3}$ spins along an edge, nearest-neighbor coupling of constant strength, and six nearest neighbors (as for a simple cubic lattice). For $J>0$ the free energy of spin-glasslike states is higher than that of corresponding states with remanent magnetization. The dependence of the coercive field $\left(B_{c}\right)$ on $J$ and $D$ is discussed and the apparent discrepancy of Chi and Alben vis $a$ vis Callen, Liu, and Cullen concerning the behavior of $B_{c}$ for large $D$ is clarified. A calculation of the temperature dependence of $B_{c}$ is presented which is reminiscent of experimental results. This random anisotropy is found to give rise to a second peak in the specific heat for suitable values of $D / J$. The magnetic susceptibility $\left(\chi_{T}\right)$ is calculated for both positive and negative $J$ and shows positive and negative paramagnetic Curie-Weiss temperatures, respectively. The slopes of the $\chi_{T}^{-1}(T)$ curves for $T$ well above the critical temperature $\left(T_{c}\right)$ have values that are roughly equal to $3 / 2$, the value appropriate to $D=0$ and $S=1$. The local order parameter $q$ is used to identify $T_{c}$, which correlates well with the critical temperature identified from other thermodynamic quantities. The presence of the random anisotropy is found to reduce $T_{c}$ by up to about $25 \%$. The results of several temperature-dependent calculations are summarized in a phase diagram and regions of paramagnetic, random ferromagnetic, and random antiferromagnetic (or spin-glass-like) behavior are identified.
\end{abstract}

\section{INTRODUCTION}

In recent years, there has been much interest in various models describing magnetism in amorphous metals. Basically, these descriptions have been proceeding along two paths. In one approach, it is assumed that the most important effect of amorphous structure on magnetism is that it in troduces fluctuations in the exchange interactions. ${ }^{1}$ In a second approach, the one considered in this paper, the crucial feature is assumed to be the existence of local-random-anisotropy fields. ${ }^{2}$ Of course, the problem of describing an amorphous magnetic material is more complicated than just choosing one of these descriptions. For example, questions involving the nature of the interactions and the nature of local-environment effects other than anisotropy may also be asked. Also, the disorder must be sufficiently characterized to allow unique calculations to be made; yet, it is often unclear how well this characterization will describe a real material. ${ }^{3}$ In addition, an amorphous solid has no translational symmetry and many of the traditional calculational procedures of solid-state physics are not applicable.

The formulation of any model for an amorphous system contains, as an essential idea, some con- cept of disorder. Therefore, a short discussion of the general concept of disorder may be appropriate. In magnetic systems, disorder can arise in several ways. ${ }^{3,4}$ One example is that of a crystallline alloy in which there are two types of atoms (say, $A$ and $B$ ) with at least one being "magnetic." This is an example of a site-disorder problem in that the bond between any two sites $(A A, B B$, or $A B)$ is determined by the state of occupancy of each site. A second example of a disordered magnetic system is that of an amorphous structure containing magnetic ions. To describe such a system, one often uses a bond-disorder model. For example, suppose one wishes to describe an amorphous magnet having only one type of atom. The simplest bond-disorder problem arises by assuming nearest-neighbor (nn) bonds (which may even be considered as fixed in number) that are statistically independent and whose strength is specified by some probability distribution. Another possible type of disorder in amorphous systems is that in which all magnetic ions are the same but reside in different environments which are characterized by different anisotropies. ${ }^{2}$ Of course, for an amorphous system, even more complicated forms of disorder are easily imagined. This paper primarily will deal with the case in which all magnetic ions the same, 
and in which the disorder arises from a random anisotropy characterized by a probability distribution.

Even supposing we may characterize an actual amorphous magnetic solid by giving some probability distribution for a fluctuating quantity [such as the magnitude of nearest-neighbor (nn) bonds or the direction of a uniaxial anisotropy of constant strength], there is still a decision to be made. We need to say whether we are considering the randomness as spatially frozen or whether we allow some spatial correlation in the randomness if this correlation results in a lowering of the free energy. ${ }^{5}$ The former case is called the quenched case and, if the bonds were the fluctuating quantity, this approach considers the bonds of particular pairs as random but fixed independent of the temperature. The later is called the annealed case and, in the bond example, this approach still assumes all bonds in the solid were chosen by the same probability distribution as for the quenched case but now allows them to arrange themselves in space so as to give the lowest free energy. We only consider the quenched case because it seems to most nearly correspond to the usual experimental situation.

In recent years it has been realized that disorder in, for example, model systems that would be ferromagnetic without the disorder, may result in more drastic changes than a lowering of the Curie temperature $\left(T_{C}\right)$ or a reduction of the magnetization at temperatures below $T_{C}$. The disorder may become so severe as to cause the magnetization for a range of temperatures below some temperature $T_{f}$ to be zero, but nevertheless the system may retain a type of magnetic order in this temperature range. One way to understand this is to imagine that below $T_{f}$ the spins are frozen in random directions, a phase which is neither paramagnetic nor ferromagnetic. ${ }^{6}$ More generally, such a phase can be characterized as one in which a long-range-order parameter vanishes and a local-order parameter, chosen to measure the degree to which each spin is frozen in orientation, does not vanish. Such a phase has been given the name spin glass. ${ }^{7}$

Alloys of noble metals (e.g., $\mathrm{Ag}, \mathrm{Au}$ ) with a few atomic percent admixture of transition metals (e.g., $\mathrm{Mn}, \mathrm{Fe}$ ) have shown behavior which has caused them to be called spin glasses. ${ }^{7}$ In such alloys, the magnetic susceptibility shows a cusp at a particular temperature $T_{f}^{\prime}$ (which would tend to indicate some kind of ordering) and neutron scattering indicates the absence of any long-range order below $T_{f}^{\prime}$. Remanence, which decreases more or less logarithmically with time, has been seen in the magnetization below $T_{f}^{\prime}$. The specific heat seems to be linear in temperature at low temperatures and does not show a well-defined peak at $T_{f}^{\prime}$, but rather a broad maximum which occurs at a temperature somewhat higher than $T_{f}^{\prime}{ }^{8}$ It is not completely clear that experimental examples of spin glasses correspond to the characterization of a spin glass as given in the preceding paragraph. ${ }^{7}$ (It is also not clear that $T_{f}^{\prime}$ characterizes exactly the same phenomena as does the $T_{f}$ of theoretical models.) Also, because the specific heat does not show a well-defined transition temperature, one might wonder if the transition to a spin-glass "phase" is really a phase transition. Other problems, which will be mentioned later, remain in attempting to construct simple models which account for all of the characteristic experimental features of spin glasses.

The models of amorphous magnetism which consider fluctuating bond $\mathbf{s}^{1,9,10}$ have been used to describe spin-glass behavior. These models are based on a relatively simple idea. For example, suppose that the bonds (or exchange interactions $J)$ arise from an Ruderman-Kittel-Kasuya-Yosida (RKKY) type interaction, then in an amorphous metal [or perhaps in crystalline alloys like $\mathrm{Cu}(\mathrm{Mn})$ ] we can see how the $J$ 's may fluctuate, even in sign. ${ }^{11}$ At low temperatures, the molecular field at a site will be nonzero and the spins will freeze into an orientation along this field. If the molecular fields at different sites are randomly oriented, then the average moment, associated with many sites, may be small or even zero. When the J's are assumed to fluctuate with a Gaussian distribution, the model is called the Edwards-Anderson ${ }^{1}$ (EA) model. Using this model, EA did a classical (basically mean-field) calculation to demonstrate that there could be a cusp in the magnetic susceptibility even when there was no overall longrange order. Shortly thereafter, Sherrington and Southern ${ }^{9}$ and Fischer ${ }^{10}$ did the corresponding quantum-mechanical calculation (Sherrington and Southern generalized the original EA model to allow for the possibility that the average of the $J$ 's was not zero). All of these treatments predicted a cusp in the magnetic specific heat which is not seen experimentally. Later, Monte Carlo calculations on EA-like Ising systems were made by Binder and Schröder ${ }^{12}$ who obtained a magnetic specific heat which better agreed with experiment. However, all of these calculations predicted that the magnetic field necessary to produce a rounding in the cusp of the magnetic susceptibility was greater than that seen experimentally. Recently, Walker and Walstedt ${ }^{13}$ have done computer model calculations of dilute randomly distributed classical spins on a face-centered-cubic lattice with 
RKKY interactions。Their results give a satisfactory description of the approximate linear lowtemperature specific heat. Luttinger ${ }^{14}$ has done a mean-field "random-site" calculation and obtains a transition to an apparent spin-glass phase (for appropriate values of the parameters). He finds a cusp in the susceptibility as well as a discontinuity in the specific heat.

The second approach that has been considered as a possibility for describing amorphous magnetism (including spin-glass behavior) is the random anisotropy model..$^{2,15,16}$ In this model, the fluctuations in exchange interaction are ignored, but a site-dependent uniaxial anisotropy term is added whose strength remains constant but whose orientation varies randomly from site to site. This model has been used in an attempt to interpret $^{15}$ the reduction of the Curie temperature and magnetization (with respect to the crystalline state) of $-\mathrm{TbFe}_{2}$ as well as to account for the large coercive forces observed. However, it seems questionable whether such a model can show a phase transition to an equilibrium spin-glass state for positive exchange. In view of the results of Chi and Alben, ${ }^{17}$ Harris and Sung, ${ }^{18}$ and the authors, ${ }^{19}$ it would appear that the spin-glass state originally predicted by Harris and Zobin ${ }^{15}$ is metastable in that it has a higher free energy than the random ferromagnetic state..$^{20}$ Of course, such a metastable state could be experimentally significant. Mention should also be made of the classical, zero-temperature mean-field calculation of Callen, Liu, and Cullen, ${ }^{21}$ whose predictions for the remanence and coercivity agree with neither Harris and Zobin ${ }^{15}$ nor Chi and Alben. ${ }^{17}$ Related calculations for the random anisotropy model have also been done by $\mathrm{Darby}^{22}$ and Richards. ${ }^{23}$

In this paper, we consider the random anisotropy model in a local-mean-field approximation, thereby treating site-to-site fluctuations in the mean field without further approximation. In Sec. II, the random anisotropy model is presented and a description of our assumptions, approximations, and calculational technique is given. In Secs. III and IV our results and conclusions are given. The major results of this work are as follows: (i) the effect of random anisotropy on the temperature dependence of several thermodynamic quantities is given; (ii) the apparent discrepancy found in the results of Chi and Alben vis $\grave{a} v i s$ those of Callen, Liu, and Cullen concerning the behavior of the coercive field for large $D$ is clarified; and (iii) a magnetic phase diagram for the equilibrium. state is constructed, and regions of paramagnetic, random ferromagnetic, and random antiferromagnetic (or spin-glass) behavior are identified.

\section{MODEL STUDIED}

\section{A. Model Hamiltonian and approximations}

We assume the following Hamiltonian:

$$
H=H_{x}+H_{a}+H_{z},
$$

where $H_{x}$ is the isotropic Heisenberg exchange

$$
H_{x}=-\frac{1}{2} \sum_{i, j} J_{i j} \vec{S}_{i} \cdot \vec{S}_{j}, \quad J_{i j}=J_{j i}, \quad J_{i i}=0,
$$

$H_{a}$ is a sum of single-ion uniaxial anisotropy terms

$$
H_{a}=-D \sum_{i}\left(\hat{k}_{i} \cdot \overrightarrow{\mathrm{S}}_{i}\right)^{2}, \quad D>0,
$$

and $H_{z}$ represents the Zeeman-interaction energy

$$
H_{z}=-\overrightarrow{\mathrm{B}} \cdot \sum_{i} \overrightarrow{\mathrm{S}}_{i} .
$$

Here $\vec{S}_{i}$ is the spin operator associated with site $i$ and $J_{i j}$ labels the exchange coupling between sites $i$ and $j$. The exchange coupling may be thought of as arising from either a direct ${ }^{24}$ or an indirect ${ }^{11}$ interaction. The magnetic anisotropy term can arise from the interaction of the gradient of the crystalline electric field with the quadrupole moment of the change distribution of, say, a rareearth ion. ${ }^{16,25}$ This term can also arise when the orbital angular momentum of the ground state is quenched. In this case, the spin-orbit term mixes an orbital contribution back into the ground state and a Hamiltonian such as Eq. (3) can be appropriate for local uniaxial anisotropy. ${ }^{25}$ The $\left\{\hat{k}_{i}\right\}$ are unit vectors which determine the local easy directions and $D$ is the strength of the anisotropy. We have assumed that the random nature of the lattice causes the local easy directions to vary randomly, but we have ignored fluctuations in the strength of the local anisotropy by making $D$ a constant, ${ }^{26}$ which is an admittedly oversimplified picture. ${ }^{16}$ We assume $S=1$, which is the smallest $S$ value for which $H_{a}$ will make a dynamical contribution. Of course, higher spin values are appropriate for rare-earth ions. Equation (4) represents the Zeeman contribution to the energy with $\vec{B}$ as the externally applied magnetic field. We have assumed an isotropic $g$ factor for simplicity. A discussion of how to convert our Hamiltonian to units that are experimentally useful is given in Appendix A.

Inorder to make this Hamiltonian tractable, we make a local-mean-field approximation (LMFA) on $H_{x}$. A derivation of this approximation from the principle of the free-energy minimum is summarized in Appendix B. It is important to note that the presence of the anisotropy term does not inhibit us from making the LMFA. We assume 
nearest-neighbor ( $\mathrm{nn}$ ) coupling only and a constant coordination number $Z=6$ (as for a simple cubic lattice). In the LMFA, the Hamiltonian becomes

$$
H_{0}=\sum_{j=1}^{N} h_{j}+C(T),
$$

where

$$
h_{j}=-\sum_{i(\text { nn to } j)} J_{i j}\left\langle\overrightarrow{\mathrm{S}}_{i}\right\rangle \cdot \overrightarrow{\mathrm{S}}_{j}-D\left(\hat{k}_{j} \cdot \overrightarrow{\mathrm{S}}_{j}\right)^{2}-\overrightarrow{\mathrm{B}} \cdot \overrightarrow{\mathrm{S}}_{j}
$$

and

$$
C(T) \equiv \frac{1}{2} \sum_{i j} J_{i j}\left\langle\overrightarrow{\mathrm{S}}_{i}\right\rangle \cdot\left\langle\overrightarrow{\mathrm{S}}_{j}\right\rangle .
$$

$C(T)$ corrects for double counting of the exchange terms when calculating the mean energy. If $A$ is some operator, by $\langle A\rangle$ we mean

$$
\langle A\rangle=Z^{-1} \operatorname{Tr}\left(e^{-\beta H_{0}} A\right),
$$

where

$$
Z=\operatorname{Tr}\left(e^{-\beta H} 0\right)
$$

is the partition function for a canonical ensemble, $\beta=1 / k T, k$ is Boltzmann's constant, and $T$ is the temperature. The trace is taken over the $3^{N}$-dimensional space appropriate for the $N$ spin-one ions. If $A=B_{j}$ only operates on the subspace appripriate to a single spin $S_{j}$, then Eqs. (8) reduce to

$$
\left\langle B_{j}\right\rangle=Z_{j}^{-1} \operatorname{Tr}\left(e^{-\beta h_{j} B_{j}}\right),
$$

where the single-site partition function is

$$
Z_{j}=\operatorname{Tr} e^{-\beta h_{j}},
$$

and the traces only need to be taken over the appropriate three-dimensional subspace.

\section{B. Evaluation of thermodynamic quantities}

We restrict ourselves to spin-one ions and chose a representation for which the $S_{j}^{2}$ and $S_{z j}$ are diagonal

$$
\begin{aligned}
& S_{j}^{2}\left|m_{j}\right\rangle=2\left|m_{j}\right\rangle, \\
& S_{z j}\left|m_{j}\right\rangle=m_{j}\left|m_{j}\right\rangle, \quad m_{j}=1,0,-1 .
\end{aligned}
$$

For each site, we define a site matrix $M^{j}$, which . has elements

$$
M_{m_{j}, m_{j}^{\prime}}^{j}=\left\langle m_{j}\left|h_{j}\right| m_{j}^{\prime}\right\rangle,
$$

eigenvalues $E_{\mu j}$, and eigenvectors (in the same representation) whose components are $\left\langle m_{j} \mid \mu_{j}\right\rangle$, where $\left|\mu_{j}\right\rangle$ is an eigenket of $h_{j}$. Using the representation for which $M^{j}$ is diagonal, the needed traces can be evaluated. Since the $\left|\mu_{j}\right\rangle$ are given by

$$
\left|\mu_{j}\right\rangle=\sum_{m_{j}}\left|m_{j}\right\rangle\left\langle m_{j} \mid \mu_{j}\right\rangle,
$$

if $B_{j}$ is a single-site operator, we have

$$
\begin{aligned}
\operatorname{Tr}\left(e^{-\beta h_{j}} B_{j}\right)= & \sum_{\mu_{j} m_{j^{\prime}} m_{j}^{\prime}} e^{-\beta E_{\mu_{j}}} \\
& \quad \times\left\langle m_{j}\left|B_{j}\right| m_{j}^{\prime}\right\rangle\left\langle m_{j}^{\prime} \mid \mu_{j}\right\rangle\left\langle m_{j} \mid \mu_{j}\right\rangle^{*} .
\end{aligned}
$$

Note that the site matrix $M^{j}$ is a function of $J \sum_{i(\text { nn to } j)}\left\langle\vec{S}_{i}\right\rangle, D, \hat{k}_{j}$, and $B$. Hence, its eigenvalues and eigenvectors are determined by these same quantities.

The relevant thermodynamic quantities can now be written down. The quantum statistical averages of the spins are

$$
\left\langle\overrightarrow{\mathrm{S}}_{j}\right\rangle=Z_{j}^{-1} \operatorname{Tr}\left(e^{-\beta h_{j}} \overrightarrow{\mathrm{S}}_{j}\right) \text {. }
$$

Because $D \neq 0$, one cannot assume that any of the components of $\left\langle\vec{S}_{j}\right\rangle$ vanish, nor that $\left\langle\vec{S}_{i}\right\rangle=\left\langle\vec{S}_{j}\right\rangle$ for $i \neq j$. Therefore, because the $h_{j}$ depend on the $\left\langle\vec{S}_{i}\right\rangle$ belonging to the nn's of site $j, \mathrm{Eq}$. (14) represents $3 N$ coupled equations. These equations are solved (with periodic boundary conditions) without further fundamental approximations by iteration and a self-consistent set of the $N\left\langle\overrightarrow{\mathrm{S}}_{j}\right\rangle$ 's are obtained. This procedure limits us to fairly small $N$. Our objective is to obtain information about the set $\left\{\left\langle\vec{S}_{j}\right\rangle\right\}$ and the other thermodynamic quantities as a function of $\beta, D, J$, and $\overrightarrow{\mathrm{B}}$. The use of the dimensionless parameters $t \equiv 1 / \beta D, j \equiv J / D$, and $b \equiv \overrightarrow{\mathrm{B}} / D$ is convenient. For example, as shown in Appendix $\mathrm{A}$, the thermodynamic quantities we calculate either depend solely on $t, j$, and $b$, or scale with $D$ for fixed $t, j$, and $b$.

Once the $\left\langle\vec{S}_{j}\right\rangle$ are obtained self-consistently, the other thermodynamic quantities can be readily written. The magnetization per spin is defined as

$$
\overrightarrow{\mathrm{m}}=\frac{1}{N} \sum_{i=1}^{N}\left\langle\overrightarrow{\mathrm{S}}_{i}\right\rangle
$$

the order parameter $q$, which measures the extent to which spins are locally "frozen" in orientation, is defined $\mathrm{as}^{7}$

$$
q=\frac{1}{N} \sum_{i=1}^{N}\left\langle\overrightarrow{\mathrm{S}}_{i}\right\rangle \cdot\left\langle\overrightarrow{\mathrm{S}}_{i}\right\rangle ;
$$

the free energy $(f)$ per spin is

$$
f=\frac{1}{N}(-k T \ln Z)=\frac{-k T}{N} \sum_{j} \ln Z_{j}+\frac{C(T)}{N},
$$

the internal energy per spin $(u)$ is

$$
u=\frac{\left\langle H_{0}\right\rangle}{N}=\frac{1}{N} \sum_{j=1}^{N}\left\langle h_{j}\right\rangle+\frac{C(T)}{N},
$$

and the entropy per spin is

$$
s=(u-f) / T \text {. }
$$

In Eq. (18), $\left\langle h_{j}\right\rangle$ can be computed from the self- 
consistent $\left\{\left\langle S_{j}\right\rangle\right\}$ which determine the eigenvalues of the corresponding site matrix. Within the LMFA, the nn correlation function is given by

$$
C_{12}=\frac{1}{N} \sum_{i=1}^{N}\left(\frac{1}{6} \sum_{j(\text { nnto } i)}\left\langle\overrightarrow{\mathrm{S}}_{i}\right\rangle \cdot\left\langle\overrightarrow{\mathrm{S}}_{j}\right\rangle\right) \text {. }
$$

The specific heat per spin at constant field $\left(C_{B}\right)$ and the magnetic susceptibility per spin at constant temperature $\left(\chi_{T}\right)$ can be numerically obtained directly by use of Eqs. (15) and (18)

$$
C_{B}=\Delta u / \Delta T=k \Delta u / \Delta(1 / \beta)
$$

and

$$
\chi_{T}=\Delta m_{z} / \Delta B_{z},
$$

where $\Delta B_{z}$ and $\Delta T$ are numerically very small and $|\overrightarrow{\mathrm{m}}| \equiv m$. We also evaluate the mean square energy fluctuations

$$
C_{B}^{(1)}=k \beta^{2}\left(\left\langle H_{0}^{2}\right\rangle-\left\langle H_{0}\right\rangle^{2}\right) \text {. }
$$

In Eq. (23), if $H_{0}$ is replaced by $H$, and $e^{-\beta H_{0}}$ is replaced by $e^{-\beta H}$, then $C_{B}^{(1)}$ would be the exact specific heat. One can also show that $C_{B}^{(1)}=C_{B}$ when all $\left\langle\overrightarrow{\mathrm{S}}_{j}\right\rangle=0$. Thus, in some sense, $C_{B}^{(1)}$ is also a measure of the specific heat. The presence of more than one specific heat, depending on the way the approximations are made, is not, of course, unique to our calculation. ${ }^{27}$

\section{Calculational techniques and summary of assumptions and approximations}

The basic calculational problem is the iteration of Eq. (14) to obtain a self-consistent set of solutions $\left\{\left\langle\vec{S}_{\boldsymbol{i}}\right\rangle\right\}$ for a given set of $\left\{\hat{k}_{\boldsymbol{i}}\right\}$ with fixed $t$, $j$, and $\vec{b}$. The $\left\{\hat{k}_{i}\right\}$ were chosen to have random directions uniformly distributed over the unit sphere. Essentially two types of calculations were made. One set of calculations, the "temperature runs," were done with fixed $j$ and $b$ (typically, $\left.b \lesssim \frac{1}{100}|j|\right)$ and varying $t$. We found that by starting at temperatures $t$ well above $t_{c} \equiv k T_{C} / D$, we were less likely to obtain metastable states. (In the body of this paper we use the subscripts $c$ and $C$ to denote any critical temperature including the Curie temperature.) To start our temperature runs at $t>t_{c}$, we had to start at $t>4|j|$ because for $D \neq 0, t_{c}$ varies from about $3 j$ to $4 j$. (For $D=0, k T_{C}=4|J|$ as shown by Appendixes C and D.) The initial values for the $\left\{\left\langle\vec{S}_{i}\right\rangle\right\}$ were chosen to be $\left\langle\vec{S}_{i}\right\rangle=\epsilon_{i} \hat{k}_{i}$, where $\epsilon_{i}= \pm 1$ is chosen by the prescription given in Appendix $\mathrm{E}$ (which outlines an initiation procedure which is not necessary in the temperature runs because of the rapid and unique convergence at high $t$, but it was important in the "field runs" described below). We iterated through the lattice and at each site evaluated the eigen- values and eigenvectors of the corresponding site matrix $M^{j}$. From these, we calculated a new value for the thermal average $\left\langle\vec{S}_{j}\right\rangle$, evaluated $\Delta_{j} \equiv\left|\left\langle\vec{S}_{j}\right\rangle^{\text {new }}-\left\langle\vec{S}_{j}\right\rangle^{\text {old }}\right|$, and replaced $\left\langle\vec{S}_{j}\right\rangle^{\text {old }}$ by $\left\langle\vec{S}_{j}\right\rangle^{\text {new }}$ in the set $\left\{\left\langle\vec{S}_{j}\right\rangle\right\}$. After one complete pass through the lattice, $\Delta=(1 / N) \sum_{i=1}^{N} \Delta_{i}$ was evaluated. The iteration procedure was continued until $\Delta$ was less than a number $E_{N \Delta}$ which was typically chosen to be the order of $10^{-5}$. It should also be emphasized that at each site $j$ in the iteration the latest available thermal averages of spins for determining $M^{j}$ were used. After a self-consistent solution was obtained, the temperature was reduced to $t-\delta$ and the procedure was repeated using as our initiating set of $\left\{\left\langle\vec{S}_{j}\right\rangle\right\}$ at $t-\delta$, the selfconsistent set $\left\{\left\langle\overrightarrow{\mathrm{S}}_{j}\right\rangle\right\}$ which we found at $t$. As expected, the convergence of this procedure was slowest for temperatures near $t_{c}$. The number of passes needed for convergence depended on the value of $t, N$, and $E_{N \Delta}$. Typically, for $t \sim t_{c}, 100$ or more passes might be needed for $N=64$, whereas for $t \gg t_{c}$, often three or four passes might be sufficient.

The other type of calculation, the field run, was similar, except that we set $t$ equal to a constant, usually much less than $t_{c}$, and varied $b_{z}$ in such a way as to trace through a hysteresis loop. $b_{z}$ was usually started near zero.

The major complication of our calculations was that for $t<t_{c}$, more than one nonvanishing set of $\left\langle\vec{S}_{j}\right\rangle$ 's which satisfied Eq. (14) was found. As shown in Appendix B, Eq. (14) results from extremizing the free energy, but different sets of solutions of Eq. (14) could correspond to different local extrema in the free energy. It seems likely that these local extrema are in fact local minima in the free energy. If $D=0$ and $T=0$, the nonvanishing solutions must correspond to the $\left\langle\vec{S}_{j}\right\rangle$ 's pointing along the molecular field-an energy minimum. More generally, by using our iteration procedure, convergence on maxima solutions seems unlikely. Where more than one solution set $\left\{\left\langle\vec{S}_{j}\right\rangle\right\}$ was found, the set with lower free energy was viewed as the more stable set. Those solutions with higher free energy may approximate physically realizable, but at best metastable states.

Finally, we summarize the assumptions and approximations which went into our model calculations: (i) the spins were coupled by a constant nearest-neighbor Heisenberg exchange interaction and experienced a uniaxial anisotropy of constant magnitude and random orientation; (ii) only $S=1$ spins were considered; (iii) periodic boundary conditions on a system with $N^{1 / 3}$ spins along an edge were used; (iv) the topology was preserved in the sense that we assumed the num- 
ber of nn's (six for our case) was constant; (v) the LMFA was used to represent the exchange coupling; (vi) small systems with $N$ much less than Avogadro's number were large enough to provide useful information. In most of our calculations, $N=64$ or 216 , but some results were obtained for $N=1000$; (vii) a practical definition of convergence $\left(\Delta<E_{N}\right)$ of the iteration procedure was adequate; and (viii) solutions could be found whose properties differ negligibly from a solution for which the free energy was either an absolute minimum or at least a local minimum of sufficient depth that, for all practical purposes, the solution was stable.

\section{RESULTS}

(a) Figures 1-3 show some typical hysteresis curves. For all curves, $B_{x}$ and $B_{y}$ were very small but not zero (of order $10^{-4}$ or less). These three figures illustrate how the coercive field increases as $j$ is decreased. We found for $j \geq 0.13$, the value appropriate to Fig. 1 , that the coercive field became very small. We also found that large coercive fields were only obtained for $D / Z J$ well above unity and these were about $1.2 \mathrm{~J}$ at low $T$.

(b) Figures 1-3 also illustrate that the remanence decreases as $D$ increases. This is physically what we would expect for random $\left\{\hat{k}_{i}\right\}$.

(c) Different initial $m_{z}\left(B_{z}\right)$ curves (virgin curves) are possible depending on the initiation procedure and/or the choice of the set of $\left\{\hat{k}_{i}\right\}$ 's. However,

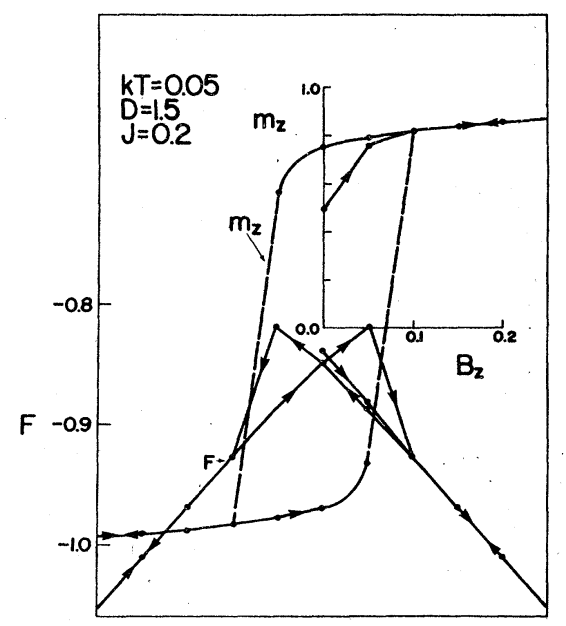

FIG. 1. Magnetization and free-energy hysteresis loops for $j=0.13$. Here $F=f+D\left\langle\sin ^{2} \theta_{i}\right\rangle_{\text {av }}, \theta_{i}$ being the angle $\hat{k}_{i}$ makes with the $z$ axis and $f$ being the free energy per spin. For a truly random set of $\hat{k}_{i},\left\langle\sin ^{2} \theta_{i}\right\rangle_{\text {av }}$ $=\frac{1}{3}$. Here $N=216$ and $N\left(E_{N \Delta}\right)=0.0005$. Note that the free energy corresponding to states on the virgin curve is higher than the free energy corresponding to states on hysteresis loop with positive magnetization.

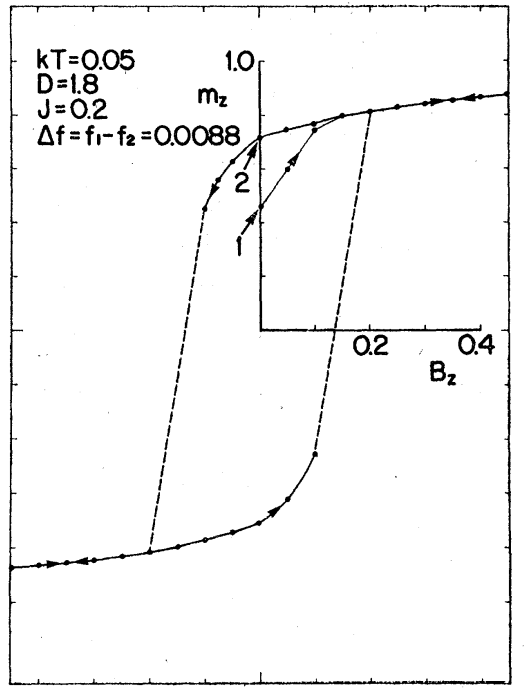

FIG. 2. Hysteresis loop for $j=0.11$. The coercive field is larger than in Fig. 1 because $j^{-1}$ is larger. Note that $f_{1}>f_{2}$. Here $N=216$ and $N\left(E_{N \Delta}\right)=0.0005$.

our experience has been that as we increase $B_{z}$ and then reduce it to zero, we are left in an $m_{z} \neq 0$ state (for $0<j<0.13$ ) that has lower free energy than that of the corresponding point on the virgin curve (see, e.g., Fig. 2). For a given $D, J$, and $T$, we have calculated many different virgin curves; yet, the corresponding hysteresis loops tended to be relatively unique. Sometimes the virgin curves had not only a small $m_{z}$ (at $B \cong 0$ ) but also a small $m$ [see, e.g., Fig. 3 , the $m(B=0)$ value on the virgin curve is $<0.2]$. A state corresponding to such a point would probably be characterized as a metastable spin-glass-like state. Note also in Fig. 3 that as $B_{z}$ is increased a sharp break occurs at $B_{z} \cong 0.15$. Harris et al. ${ }^{15}$ apparently characterized such breaks as qualitatively defining a coercive field, but we see that

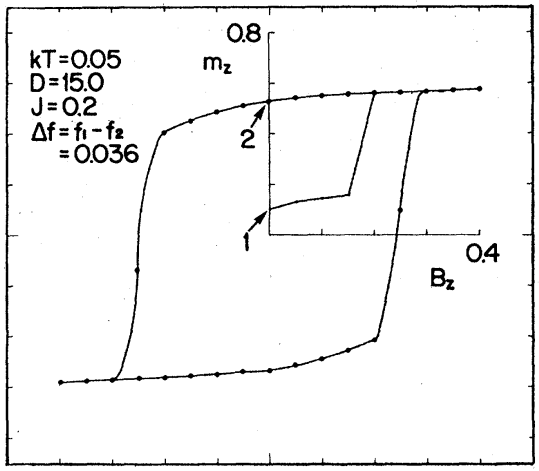

FIG. 3. Hysteresis loop for $j=0.013$. The large coercive field is due to the small value of $j$. Note that $f_{1}>f_{2}$. Here $N=64$ and $N\left(E_{N \Delta}\right)=0.0001$. 
a more precise characterization is obtained from the full hysteresis loop.

(d) $B_{c}$ was essentially zero for all cases with $J<0$ that we investigated.

(e) The hysteresis curves are for thermodynamic "equilibrium" (or quasiequilibrium in the sense that the solutions $\left\{\left\langle\vec{S}_{j}\right\rangle\right\}$ are obtained when the free energy is an extremum in the space of the $\left.\left\{\left\langle\vec{S}_{j}\right\rangle\right\}\right)$. If $D$ is very large compared to $J$, there is a huge " $D$ barrier" for reversal of the spin at any site, but our calculation does not and should not give a coercive field proportional to the barrier. This point is discussed later.

(f) Figure 4 is included to illustrate the effect of a nonrandom exchange anisotropy on the random anisotropy model. For these curves $K$ is defined by writing the original exchange Hamiltonian as

$$
\begin{aligned}
H_{x}= & -\frac{1}{2} J \sum_{i j,(\mathrm{nn})} S_{i z} S_{i z} \\
& -\frac{1}{2} K \sum_{i j,(\mathrm{nn})}\left(S_{i x} S_{j x}+S_{i y} S_{j y}\right) .
\end{aligned}
$$

Note when $J / K$ is relatively large, the hysteresis is relatively square with a large coercive field of about 0.8 (when $K=0, B_{c}=Z J S$ at $T=0$ ) and when $J / K$ is relatively small, the coercive field is much smaller $(<0.2)$. This is what we would expect because a large $J / K$ means it is energetically favorable to magnetize in the $z$ direction whereas a small $J / K$ makes it favorable to magnetize in the $x-y$ plane. This figure is reminiscent of hysteresis data obtained by Clark ${ }^{28}$ for sputtered amorphous $\mathrm{TbFe}_{2}$ annealed in a magnetic field at $304^{\circ} \mathrm{C}$. Comparing this figure with Clark's data suggests that annealing in a basal magnetic field introduces an exchange anisotropy similar to that given by the above Hamiltonian.

(g) Figure 5 shows that the $m_{z}\left(B_{z}\right)$ curve for

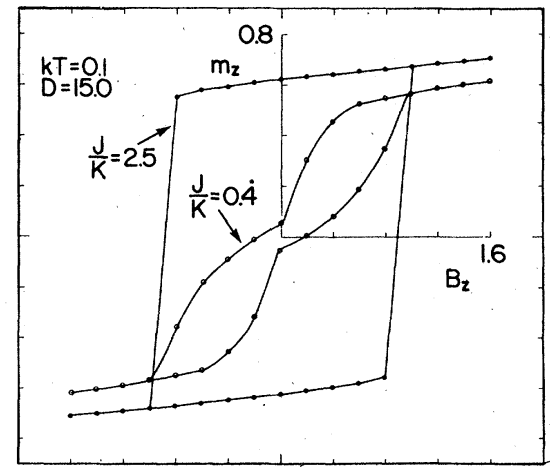

FIG. 4. Two hysteresis loops illustrating the effect of exchange anisotropy. The "square" loop has $J=0.5$ and $K=0.2$. The "bow-tie" loop has $J=0.2$ and $K=0.5$. Both loops are for $N=64$ and $N\left(E_{N \Delta}\right)=0.001$.

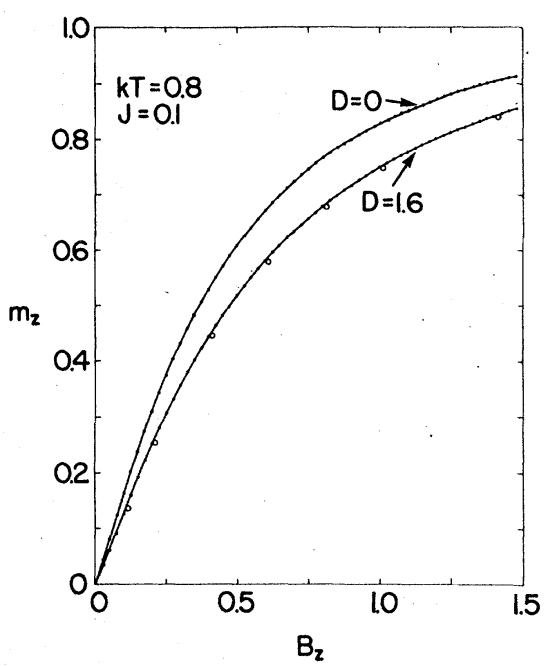

FIG. 5. $m_{z}\left(B_{z}\right)$ above the critical temperature with $D=1.6, N=216$ (lines) and $N\left(E_{N \Delta}\right)=0.0005$; and for $N=64$ (circles) and $N\left(E_{N \Delta}\right)=0.001$. There was no significant difference for $D=0$ between $N=64$ and $N=216$.

$D \neq 0$ lies below that curve for $D=0$, even when $T>T_{C}$. This could be useful in trying to establish the relevance of the random anisotropy model to an experimental situation. Of course, for sufficiently large $B_{z}$ at fixed $T$ or sufficiently large $T$ [and for all $B_{z}>0$ such that $\beta B_{z} \ll 1, \beta D \ll 1$, $\beta J \ll 1$, see Eq. (D8)], the two curves will come together.

(h) In Fig. 6, we show how the coercive field

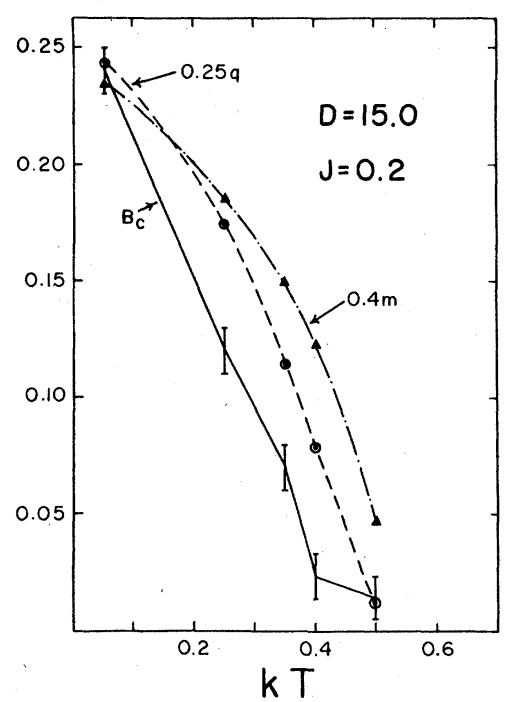

FIG. 6. $B_{c}$ (solid line), $q$ (dashed line), and $m$ vs $T$. In calculating $q$ and $m, B_{z}=0.0001$. Error bars on $B_{c}$ come from determining the coercive field from hysteresis loops by interpolation between points. Note that $B_{c}$ drops more rapidly with temperature than either $m$ or $q$. For all points, $N=64$ and $N\left(E_{N \Delta}\right) \leq 0.001$. 
varies with temperature. The rapid increase in $B_{c}$ with decreasing temperature is reminiscent of experimental measurements of the coercive field made on amorphous magnets. ${ }^{29}$ These $N=64$ results are only preliminary. We did not do a calculation for larger $N$ because each point requires a hysteresis calculation making more extensive results expensive. Note that the coercive field drops rather rapidly with temperature compared to either $m$ or $q$. This tends to indicate that a cooperative effect is rather efficient in reducing the coercive field.

(i) Figures 7-9 show typical temperature variations of the thermodynamic quantities for small $j$. As mentioned earlier, these results were obtained by starting at high temperatures (above $T_{c}$ ) and "cooling" so that any ordering which appears is not an artifact of the initiation process used for starting the iteration. Starting at low temperatures and "heating" tended to yield curves with discontinuous jumps. Such jumps also appeared in the free energy and were interpreted as due to transitions between metastable states. Because we decided to focus our investigation on the equilibrium states, this "cooling" procedure was adopted.

(j) In Fig. 7, $q$ rises from zero at a temperature we identify as $T_{c}$. For the case shown, $T_{c}$ is about $20 \%$ lower than the ordering temperature for $J=0.04$ and $D=0$ (arrow). Notice that $m$ and the nn correlation function (Fig. 9) also become appreciable below $T_{C}$.

(k) $\chi_{\boldsymbol{T}}$, shown in Figs. 7 and 8, obeys a CurieWeiss law for $S=1$ at high temperatures. By Eq. (D8) this behavior is expected, but it is perhaps

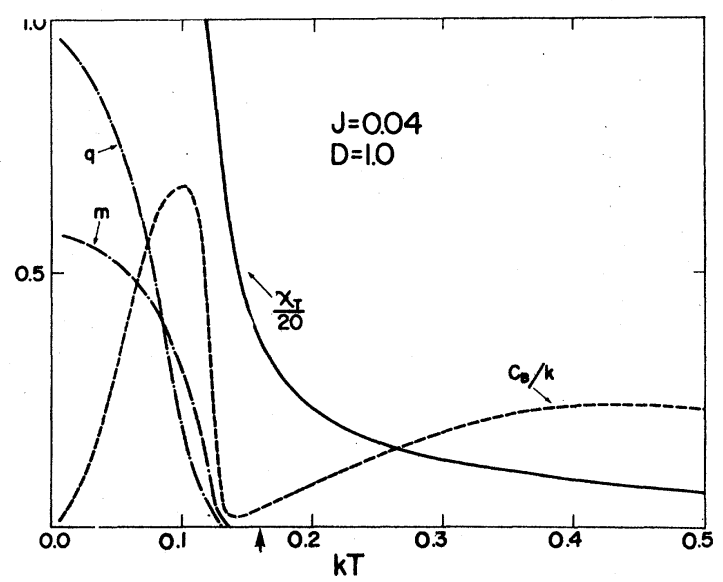

FIG. 7. Order parameter $q(T)$, magnetization $m(T)$, susceptibility $\chi_{T}(T)$, and specific heat $C_{B}(T)$. Arrow indicates ordering temperature for a system with $J=0.04$ and $D=0$. For these curves $B_{z}=0.0004, \Delta B \simeq 0.1 B_{z}$, $N=216$, and $N\left(E_{N \Delta}\right)=0.005$.

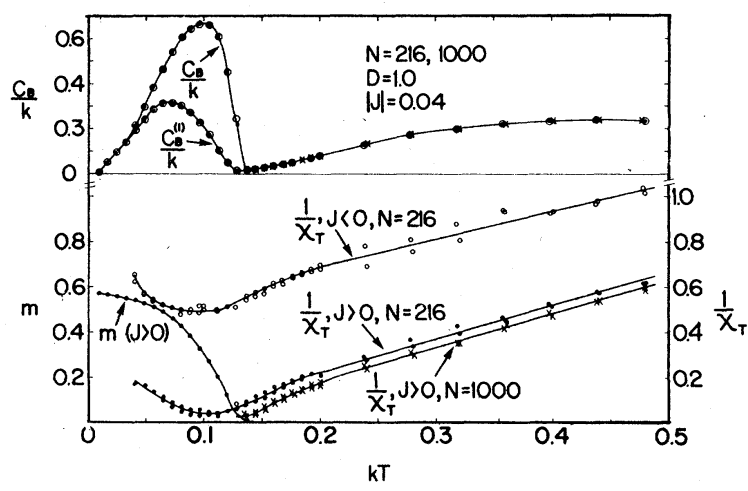

FIG. 8. $\chi_{T}^{-1}, C_{B}, C_{B}^{(1)}$, and $m$ vs $T$ for $|J|=0.04$, $N\left(E_{N \Delta}\right)=0.005$ (for $N=216$ ) or 0.01 (for $N=1000$ ), and $B_{z}=0.00038$. For $J<0, m \leq 0.02$ and it was not plotted. Results for $N=1000$ and $J>0$ are denoted by crosses, those for $N=216$ by solid dots $(J>0)$ and open circles $(J<0)$. Results for $N=1000$ were obtained only down to temperatures just above $T_{C}$. Values of $\Delta B_{z}$ used for evaluating the $\chi_{T}$ were $\sim B_{x} \simeq 10^{-5}$, which may account for the scatter in points. Position of the circles and solid dots on specific-heat curves are indistinguishable.

surprising that the linear behavior of $\chi_{\bar{T}}^{-1}$ seems to persist (see Fig. 8) to $T$ so near $T_{C}$. In fact, the $\chi_{\overline{\boldsymbol{T}}}^{-1}(T)$ curves are possibly not quite linear at these low temperatures; which would explain their failure to extrapolate to $4|J|$ as indicated by Appendix D. Certainly our temperatures are too low to guarantee the accuracy of Eq. (D8). It should be noted that if $D$ is large enough the slope of $\chi_{\bar{T}}^{-1}(T)$ is greater than $\frac{3}{2}$ for $k T \lesssim 0.2 D$.

(1) The curve for the specific heat may be the most interesting one in Fig. 7 (see also Fig. 8).

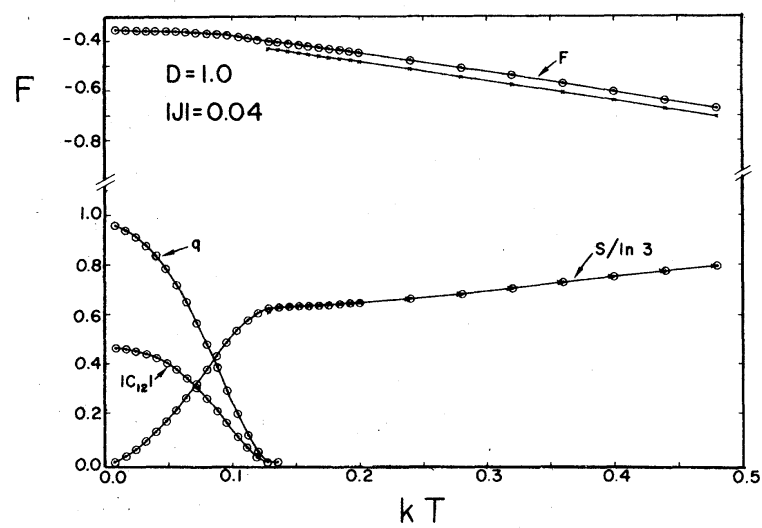

FIG. 9. $s / k \ln 3,\left|C_{12}\right|, q$, and $F$ (as in Fig. 1) vs. $T$ for $|J|, E_{N \Delta}, B_{z}$, and all symbols as in Fig. 8. Again, results for $N=1000$ were obtained only for $T>T_{C}$. Slight shift in the free energy between $N=216$ and $N=1000 \mathrm{re}$ sults can be accounted for by the fact that tie set $\left\{\hat{k}_{i}\right\}$ is different in these two cases. 
The low-temperature peak, which rises sharply at $T_{C}$, is what one would expect for a mean-field theory. The second peak is purely an effect of $D$, occurring at $k T \cong 0.44 D$. Note (Fig. 8) that $C_{B}=C_{B}^{(1)}$ for $T>T_{C}$ and $B \simeq 0$. For $T<T_{C}, C_{B}$ peaks at a higher temperature and has a more pronounced maximum than does $C_{B}^{(1)}$.

(m) Notice that for $J>0$ (Figs. 7 and 8) the magnetized $(m>0)$ solution appears rather than a spinglass-like solution $(m \approx 0)$. We believe this occurs because the system essentially was brought to thermodynamic equilibrium at each temperature before lowering the temperature.

(n) Figure 8 compares $\chi \overline{\boldsymbol{T}}^{-1}(T)$ for $J>0$ and $J<0$ (same magnitude). $m(J<0)$ is essentially zero for all $T . T_{C}$ and the thermodynamic quantities except $m$ and $\chi_{T}$ are not affected by the sign of $J$ (see Appendix C). The fact that the minima in $\chi \bar{T}^{-1}(T)$ occur at slightly different temperatures is probably due to the fact that neither $E_{N \Delta}$ nor the set of the $\left\{\hat{k}_{i}\right\}$ were the same for the two cases shown. (However, it should be noted that values of $\chi_{\boldsymbol{T}}$ very near $T_{\boldsymbol{C}}$ have no physical meaning because of effects due to the finite size of our system; however, these effects should not be important, even for $N=64$, if $k\left|T-T_{C}\right| \geq \frac{2}{3} J$, which corresponds in a simple mean-field model to the correlation length per atomic spacing being less than unity. ${ }^{24}$ )

(o) Figures 8 and 9 also compare variations in thermodynamic quantities with $N$. The $N=1000$ results are limited to $T>T_{C}$ because of the expense of this calculation. The slight shift in free energy (Fig. 9) is due to the fact that the set $\left\{\hat{k}_{i}\right\}$ are not the same.

(p) As shown in Fig. 9, $q$ remains unchanged under the $J \rightarrow-J$ transformation. Similarly $f, C_{B}$, $C_{B}^{(1)}, S$, and $\left|C_{12}\right|$ are invariant under $J \rightarrow-J$, as shown in Figs. 8 and 9.

(q) In Fig. 10 we show the results of one calculation which indicates that spin-glass-like states at low temperatures are not stable if $j z 0.12$. We have assumed that the $m<0.2$ state for our small system is somewhat analogous to a spin-glass state. It is interesting that the $j \approx 0.12$ value is very near the critical value of $j$ below which one gets sizable coercive fields.

(r) By making many temperature runs of the type in Figs. 7-9, we were able to construct the phase diagram (Fig. 11) for the equilibrium states of the Hamiltonian in Eq. (5). The curve shown should be regarded as accurate to no more than a few percent. $k T=4|J|$ represents the ordering temperature as $D \rightarrow 0$ [see Eq. (D14)]. There are three regions. The paramagnetic region $m=0$, $q=0$, the random ferromagnetic region $J>0, m \neq 0$ $q \neq 0$ and the random antiferromagnetic (or spin-

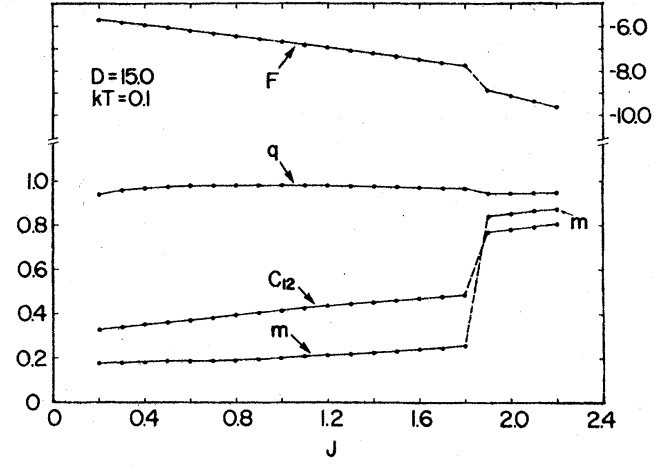

FIG. 10. $F$ (see Fig. 1), $q, C_{12}$, and $m$ vs. J. Note the transition for $j=J / D \cong 0.12$. For these curves, $B_{z}$ $=0.0001, N=64$, and $N\left(E_{N \Delta}\right)=0.001$.

glass-like) region $\delta<0, m=0, q \neq 0$. Notice that $T_{C}(j \lesssim 0.02) \simeq 0.75 T_{C}(D=0)$. As we have already indicated, evidence for metastable spin-glasslike states for positive values of $j \lessgtr 0.12$ was found, but an attempt to indicate these states by phase lines was not made because they have higher free energy than the random ferromagnetic states and appear to be nonunique in the sense that different metastable states (with highly variable $m$ ) can be reached at low temperatures (with $B \approx 0$ ) depending on the random set of $\left\{\hat{k}_{i}\right\}$ chosen and on the initiation procedure.

(s) The initial drop in $T_{C}$ with increasing $D /|J|$ agrees qualitatively with the phase diagram of Harris et al. ${ }^{15}$ but we do not obtain an appreciable subsequent rise in $T_{C}$ for large $D /|J|$. Such a rise could perhaps be accounted for in a nonequilibrium statistical calculation by allowing for a metastable state with many spins trapped by the $D$ barrier. The rise would then represent a "critical temperature" for a spin-glass-like state (as originally obtained by Harris et al.) rather than a $T_{c}$ for an equilibrium random magnetic state. Because we are doing equilibrium statistic-

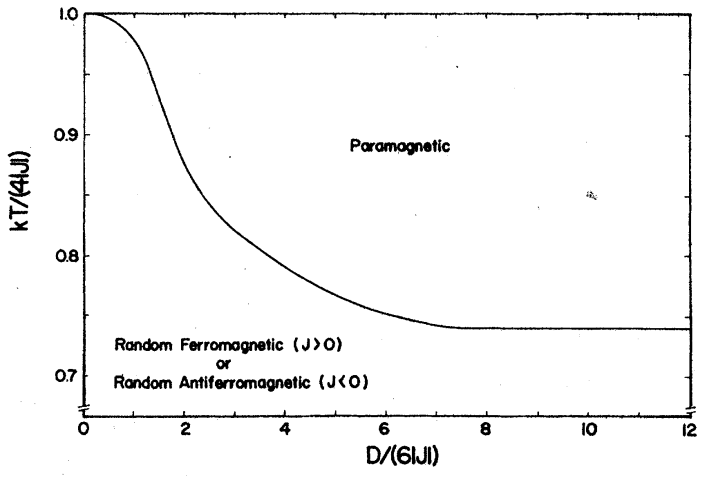

FIG. 11. Magnetic phase diagram showing effect of local anistropy strength on the ordering temperature. 
al calculations, we do not see such effects. Perhaps they were originally obtained by Harris et al. because of their approximation to the molecular field or because of insufficient convergence.

\section{DISCUSSION AND CONCLUSIONS}

Perhaps the most interesting question concerning the random anisotropy model is whether with $T$ and $j$ small enough it allows for spin-glass-like behavior for positive exchange. Our hysteresis calculations tend to confirm those of $\mathrm{Chi}$ and Alben $^{17}$ in that they support the following idea: the $\left\{\left\langle\vec{S}_{j}\right\rangle\right\}$ solutions at low $T$ and $j$ which have $q / m^{2}$ fairly large are higher in free energy (for the same values of all parameters) than those which have $O(q)=O\left(m^{2}\right)$ and which correspond to states on the hysteresis curve. By our numerical procedure, we never know if we have found all solutions, and so we cannot absolutely rule out the possibility of an equilibrium spin-glass-like solution (for $J>0$ ) which has lower free energy than all other solutions, but we believe this is unlikely. We should also mention that there is always the possibility that there are metastable spin-glasslike states that equilibrium statistical mechanics give no hint of. Nonequilibrium calculations would be needed to settle this point.

Whether one wants to call the fairly large $q^{2} / m$ solutions spin-glass-like or not, we leave open. But we do suggest if these solutions also have $C_{12}$ small (for large $N$ ), then they are more likely due to spin glassiness than to the polydomain effects which have been suggested by $\mathrm{Chi}$ and Alben. An example is given in Fig. 10 where, for $J<1.8$, $C_{12}$ is relatively small; yet, for such a small lattice, the distinction between a spin glass and a polydomain admittedly seems rather artificial.

Another interesting feature of our calculation is the qualitative effect of $j$ on various properties of the system. When $j \gtrsim 0.2$, we observe a very small, essentially vanishing, coercive field. For large $j$ we would expect the spins to be "locked" together at low temperatures and hence turn as a unit in the hysteresis cycle. Because the $\left\{\hat{k}_{i}\right\}$ 's are random, turning of the spins does not change the anisotropy energy appreciably, and so $B_{c}$ should be small. If $j$ is very small, then the spins are essentially independent. At low temperatures, we would expect $B_{c}$ to be approximately equal to the net energy required to turn one spin, and because this spin resides in an exchange field of $O(J)$, we would expect $B_{c}$ of $O(J)$. This is what our calculation yields. Notice that because we are dealing with a thermal activation from a metastable state to another state (which is probably stable), the large " $D$ barrier" between these states does not affect the coercive field. This is to be contrasted with the results of Callen $e t a l .{ }^{21}$ who find a strong $D$ dependence in the coercive field. It is possible, however, for the relaxation time [for the spin to turn over in a field of $O(J)$ ] to be very long. Then a coercive field of $O(J)$ may not be the experimentally important one. From energy considerations one would then expect a coercive field of $O(D)$. In particular, because thermal activation is not possible, at $T=0$ one would expect the result of Callen et al. to be the correct one. Our calculations also suggest that there is fairly sharp transition from a large coercive field of $O(J)$ to a small coercive field. This transition occurs at $j \simeq 0.13$ or roughly $Z j \simeq 1$. This is in qualitative agreement with $\mathrm{Chi}$ and Alben's ${ }^{17}$ zero temperature classical calculation. Thus evidently, the coercive field obtained by $\mathrm{Chi}$ and Alben is really only a possibility at finite temperature.

We already mentioned the effect of $j$ on the specific heat $C_{B}$. If $j \approx 1 / Z$, then $C_{B}$ has a fairly prominent tail for $T>T_{C}$. If $j \lesssim 2 / 3 Z$, then $C_{B}$ has two peaks, a broad one near $k T_{C} \approx 0.44 D$ and a sharper one at $k T \lesssim 4 J$. The occurence of two peaks in experimental data for $C_{B}$ on amorphous systems would be a reason to consider the random anisotropy model as appropriate for interpretation of the data. It should be mentioned that Coey and von Molnar ${ }^{30}$ have measured the low-temperature specific heat of an amorphous Dy-Cu alloy and that their data may be fitted well to the random anisotropy model for $D^{E} / k=4.5 \mathrm{~K}$ (see Appendix A for a definition of $D^{E}$ as well as a discussion on how to convert the parameters used in this chapter to those that are more useful experimentally).

We have mentioned that the susceptibility follows a Curie-Weiss law for $T$ greater than and not too near $T_{C}$. This holds true even if $|J|$ is small. Further, the slope of the $1 / \chi_{T}$ curves is fairly close to what one would get for $D=0$ provided $T$ is sufficiently large. For large $D$ and intermediate $T$, further calculations have shown slopes greater than $\frac{3}{2}$. As is expected, the $1 / \chi_{T}$ curve for $J<0$ lies above the $1 / \chi_{T}$ curve for $J>0$ corresponding to a negative paramagnetic Curie temperature in the $J<0$ case. All of these results are consistent with Appendix D, but the nearly linear behavior for $T \gtrsim T_{C}$ is not predicted (nor prohibited).

The entropy curves also reflect the effect of $D$. A large $D(>Z J)$ leaves the system partially ordered even above $T_{C}$, and the entropy $s \rightarrow k \ln 3$ only for $k T>D$.

Perhaps the most important result of our calculation is summarized in the phase diagram of Fig. 11. We believe this phase diagram is ap- 
propriate when the spins are in their true equilibrium states. In Appendix $\mathrm{C}$, which uses the LMFA applied to our system, we show that the ordering temperatures shown in the phase diagram are required to be symmetric under $J \rightarrow-J$. Since our technique of calculation did not seem to lead to unique low-temperature metastable solutions, we leave open the question of how to subdivide the random ferromagnetic region into a spin-glasslike subregion and a ferromagnetic-only subregion. Although Harris and Zobin ${ }^{15}$ made such a subdivision and our Fig. 11 suggests that this is possible, the nonuniqueness of these metastable solutions is troubling. If the nonuniqueness is only an artifact of our using small systems (since we got these different solutions with different $\left\{\hat{k}_{i}\right\}$ ), then there is no conflict. However, we suggest that the character of the metastable solutions and of their relation to actual experimental results needs to be further clarified. We should also point out that our LMFA treats the local mean field exactly (in contrast to Harris and Zobin' $\mathbf{s}^{15}$ calculation), although at the cost of limiting us to small systems. Since hysteresis and temperature variations of the thermodynamic quantities are not changed appreciably by increasing $N$ from 64 to 216 , it would be unlikely that the qualitative nature of the metastable states were strongly dependent on $N$.

\section{ACKNOWLEDGMENT}

This work was supported in part by NSF Grant No. DMR 76-17417. One of the authors (J.D.P.) would like to thank the Physics and Astronomy Department at the University of Nebraska for its hospitality during his sabbatical leave. We would like to thank R. Harris for useful discussions and R. C. Weger for his initial help with the computer programs. We are grateful to the Physics and Astronomy Department for supporting the cost of our computer calculations.

\section{APPENDIX A: CONVERSION TO PRACTICAL UNITS}

It is convenient to write the Hamiltonian of Eq. (1) as

$$
\begin{aligned}
H= & -\frac{1}{2} \sum_{i, j} J_{i, j}^{E} \vec{S}_{i} \cdot \overrightarrow{\mathrm{S}}_{j}-D^{E} \sum_{i}\left(\hat{k}_{i} \cdot \overrightarrow{\mathrm{S}}_{i}\right)^{2} \\
& -g \mu_{B} \overrightarrow{\mathrm{B}}^{E} \cdot \sum_{i} \overrightarrow{\mathrm{S}}_{i},
\end{aligned}
$$

where the superscript $E$ denotes that $J^{E}, D^{E}$, and $g \mu_{B} B^{E}$, along with $k T^{E}$, all have the units of energy. Here $\mu_{B}$ (the Bohr magneton) and $k$ (Boltzmann's constant) both have conventional units. The Lande $g$ factor $(g)$, the $\left\{\hat{k}_{i}\right\}$ and the
$\left\{\vec{S}_{i}\right\}$ are dimensionless. The reduced quantities are now defined by

$$
\begin{aligned}
& j=J / D=J^{E} / D^{E}, \\
& t=1 / \beta D=k T^{E} / D^{E}, \\
& b=B / D=g \mu_{B} B^{E} / D^{E} .
\end{aligned}
$$

Thus $j=1$ becomes $J^{E}=D^{E}, t=4 j$ becomes $T^{E}$ $=4 J^{E} / k$, and $b=1$ becomes $B^{E}=D^{E} / g \mu_{B}$.

By Eq. (18), the internal energy per spin $(u)$ has the property

$$
u=u\left(J^{E}, D^{E}, B^{E}, \beta\right)=D^{E} u(j, 1, b, t)
$$

Many of our calculations were done with $D^{E}=1$. If $D^{E} \neq 1$, we can use (A5) to determine $u$. A similar remark holds for the free energy per spin. It is also easy to show that the entropy per spin, specific heat, and $C_{B}^{(1)}[\mathrm{Eq} .(23)]$ are unaffected by changing $D^{E}$ (for fixed $j, b, t$ ).

If $\rho$ is the number of ions per unit volume, the actual magnetization is

$$
\overrightarrow{\mathrm{M}}^{a}=g \mu_{B} \rho \overrightarrow{\mathrm{m}}
$$

where $\overrightarrow{\mathrm{m}}$ is given by Eq. (15) and depends only on $j, b$, and $t$.

The corresponding isothermal susceptibility $(\chi \underset{T}{a})$ is

$$
\chi_{T}^{a}=\rho\left(g \mu_{B}\right)^{2} \chi_{T},
$$

where $\chi_{T}$ is given by Eq. (22).

The coercive force can also be shown to scale with $D$ (or $J$ ) for fixed $j$ and $t$. We know that $m_{z}=m_{k}(j, b, t)$; therefore, $0=m_{z}\left(j, b_{c}, t\right)$

or

$$
b_{c}=B_{c} / D=f(j, t)
$$

and

$$
B_{c}=D f(j, t) .
$$

Also,

$$
B_{c}=J g(j, t)
$$

where

$$
j g=f .
$$

Even the Curie temperature has a similar property, for $0=\overrightarrow{\mathrm{m}}\left(j, 0, t_{c}\right)$ or $t_{c}=h(j)$, therefore,

$$
k T_{c}=D h(j)
$$

or

$$
k T_{C}=J P(j),
$$

where

$$
h=j P \text {. }
$$


APPENDIX B: LMFA FROM THE PRINCIPLE OF THE FREEENERGY MINIMUM

If the free energy $F$ corresponding to the Hamiltonian $\boldsymbol{H}$ is

$$
F(H)=-(1 / \beta) \ln \left(\operatorname{Tr} e^{-\beta H}\right),
$$

then it can be shown that ${ }^{31}$

$$
F(H) \leqslant F\left(H_{0}\right)+\left\langle H^{\prime}\right\rangle_{0} \equiv F_{\text {mod }}(H),
$$

where mod stands for the model defined below

$$
H \equiv H_{0}+H^{\prime}
$$

and

$$
\left\langle H^{\prime}\right\rangle_{0}=\operatorname{Tr}\left(e^{-\beta H_{0}} H^{\prime}\right) / \operatorname{Tr}\left(e^{-\beta H_{0}}\right) .
$$

We wish to use the inequality (B2) to obtain an approximation for the Hamiltonian $H$ of Eq. (1). We choose $H_{0}$, which will be our approximate model Hamiltonian, by linearizing the bilinear exchange terms of $H$. Hence

$$
\begin{aligned}
H_{0} & =-\sum_{i j} J_{i j} \overrightarrow{\tilde{S}}_{i} \cdot \overrightarrow{\mathrm{S}}_{j}+C(T)-D \sum_{j}\left(\hat{k}_{j} \cdot \overrightarrow{\mathrm{S}}_{j}\right)^{2} \\
& -\overrightarrow{\mathrm{B}} \cdot \sum_{j} \overrightarrow{\mathrm{S}}_{j} \equiv \sum_{j} \tilde{h}_{j}+C(T),
\end{aligned}
$$

where $\overrightarrow{\tilde{S}}_{j}$ is a temperature-dependent vector, $C(T)$ is a temperature-dependent scalar, and

$$
\tilde{h}_{j}=-\sum_{i} J_{i j} \overrightarrow{\tilde{\mathrm{S}}}_{i} \cdot \overrightarrow{\mathrm{S}}_{j}-D\left(\hat{k}_{j} \cdot \overrightarrow{\mathrm{S}}_{j}\right)^{2}-\overrightarrow{\mathrm{B}} \cdot \overrightarrow{\mathrm{S}}_{j} .
$$

The $\left\{\vec{S}_{j}\right\}$ are chosen in such a way to extremize $F_{\text {mod }}(H)$, which is independent of $C(T), C(T)$ is chosen so that $F_{\text {mod }}(H)=F\left(H_{0}\right)$, making $H_{0}$ a logical choice for a model Hamiltonian. It should be noted that by choosing a Hamiltonian in this way, no additional consideration for double counting need be taken, e.g., $\langle H\rangle_{0}=\left\langle H_{0}\right\rangle_{0}$.

Combining Eqs. (B2)-(B5) we find

$$
\begin{aligned}
F_{\text {mod }}(H)= & -\frac{1}{\beta} \sum_{i} \ln \left(\operatorname{Tr} e^{-\beta \tilde{h}_{i}}\right) . \\
& -\frac{1}{2} \sum_{i j} J_{i j}\left\langle\overleftrightarrow{\mathrm{S}}_{i}\right\rangle_{0} \cdot\left\langle\overrightarrow{\mathrm{S}}_{j}\right\rangle_{0} \\
& +\sum_{i j} J_{i j} \overrightarrow{\tilde{\mathrm{S}}}_{i} \cdot\left\langle\overrightarrow{\mathrm{S}}_{j}\right\rangle_{0} .
\end{aligned}
$$

If $\vec{S}_{d k}$ is the $d$ th component of $\overrightarrow{\tilde{S}}_{k}$, then

$$
\frac{\partial}{\partial \widetilde{S}_{d k}} F_{\text {mod }}(H)=\sum_{j}\left(\frac{\partial\left\langle\vec{S}_{j}\right\rangle_{0}}{\partial \widetilde{S}_{d k}}\right) \cdot\left(\sum_{i} J_{i j}\left(\overrightarrow{\tilde{S}}_{i}-\left\langle\vec{S}_{i}\right\rangle_{0}\right)\right) .
$$

Clearly, a solution which extremizes $F_{\text {mod }}(H)$ is

$$
\overrightarrow{\tilde{S}}_{i}=\left\langle\vec{S}_{i}\right\rangle_{0}
$$

It is easy to see that if Eq. (B9) is satisfied, then $F\left(H_{0}\right)=F_{\text {mod }}\left(H_{0}\right)$, where $H_{0}$ is given by Eq. (5). Using Eqs. (B4) (with $H^{\prime}$ replaced by $A$, any operator of interest for our system), (B5) and (B9), we can compute in the LMFA the statistical average of $A$.

\section{APPENDIX C: INVARIANCE OF QUANTITIES UNDER $J_{i j} \rightarrow-J_{i j}$}

We assume the LMFA, $\overrightarrow{\mathrm{B}}=0$, and nn interactions with six nn's. We can break our system into two "sublattices" in which every spin of one sublattice is a nn of a spin of the other sublattice. Let these two sublattices be called $a$ and $b$. Let the corresponding molecular fields by $H_{a_{i}}$ $=\sum_{j,(\mathrm{nn})} J_{a_{i} b_{j}}\left\langle\overrightarrow{\mathrm{S}}_{b_{j}}\right\rangle$ and $\overrightarrow{\mathrm{H}}_{b_{j}}=\sum_{i} J_{a_{i} b_{j}}\left\langle\overrightarrow{\mathrm{S}}_{a_{i}}\right\rangle$, and let $\vec{A}_{a i}$ and $\overrightarrow{\mathrm{B}}_{b_{j}}$ be vector operators associated with the sites $a_{i}$ and $b_{j}$. Then for fixed $\beta, D,\left\{\hat{k}_{a i}\right.$, $\left.\hat{k}_{b_{j}}\right\}$,

$$
\left\langle\overrightarrow{\mathrm{A}}_{a_{i}}\right\rangle=\overrightarrow{\mathrm{g}}_{i}\left(\overrightarrow{\mathrm{H}}_{a_{i}}\right)=Z_{a_{i}}^{-1} \operatorname{Tr}\left(e^{-\beta_{a_{i}}} \overrightarrow{\mathrm{A}}_{a_{i}}\right),
$$

where

$$
Z_{a_{i}}=\operatorname{Tr} e^{-B h_{a i}}
$$

and

$$
\left\langle\overrightarrow{\mathrm{B}}_{b_{j}}\right\rangle=\overrightarrow{\mathrm{h}}_{j}\left(\overrightarrow{\mathrm{H}}_{b_{j}}\right)=Z_{b_{j}}^{-1} \operatorname{Tr}\left(e^{-\beta h_{b j}} \overrightarrow{\mathrm{B}}_{b_{j}}\right),
$$

where

$$
Z_{b_{j} .}=\operatorname{Tr} e^{-\beta_{b_{j}}},
$$

and where

$$
\begin{aligned}
& h_{a_{i}}=-\overrightarrow{\mathrm{H}}_{a_{i}} \cdot \overrightarrow{\mathrm{S}}_{a_{i}}-D\left(\hat{k}_{a_{i}} \cdot \overrightarrow{\mathrm{S}}_{a_{i}}\right)^{2}, \\
& h_{b_{j}}=-\overrightarrow{\mathrm{H}}_{b_{j}} \cdot \overrightarrow{\mathrm{S}}_{b_{j}}-D\left(\hat{k}_{b_{j}} \cdot \overrightarrow{\mathrm{S}}_{b_{j}}\right)^{2} .
\end{aligned}
$$

At the site $a_{i}$ (or $b_{j}$ ), if we let $\overrightarrow{\mathrm{H}}_{a_{i}}--\overrightarrow{\mathrm{H}}_{a_{i}}$ (or $\overrightarrow{\mathrm{H}}_{b_{j}}--\overrightarrow{\mathrm{H}}_{b_{j}}$ ), we can reproduce the original physical situation at that site by an inversion of the coordinate system at that site. Thus

$$
\begin{aligned}
& \overrightarrow{\mathrm{g}}_{i}\left(-\overrightarrow{\mathrm{H}}_{a_{i}}\right)=-\overrightarrow{\mathrm{g}}_{i}\left(\overrightarrow{\mathrm{H}}_{a_{i}}\right), \\
& \overrightarrow{\mathrm{h}}_{j}\left(-\overrightarrow{\mathrm{H}}_{b_{j}}\right)=-\overrightarrow{\mathrm{h}}_{j}\left(\overrightarrow{\mathrm{H}}_{b_{j}}\right) .
\end{aligned}
$$

It also follows that the statistical average of a site dependent scalar operator would be left invariant to a change in sign of the molecular field.

From the above we can say that if $\left\langle\mathbb{S}_{a_{i}}\right\rangle^{(+)}$and $\left\langle\overrightarrow{\mathrm{S}}_{b_{j}}\right\rangle^{(+)}$are self-consistent solutions for all $\mathrm{nn}$ $\left.J_{a_{i} b_{j}} \equiv J_{a_{i} b_{j}}^{(+)}\right\rangle 0$, then $\left\langle\overrightarrow{\mathrm{S}}_{a_{i}}\right\rangle^{(-)}=\left\langle\overrightarrow{\mathrm{S}}_{a_{i}}\right\rangle^{(+)}$and $\left\langle\overrightarrow{\mathrm{S}}_{b_{j}}\right\rangle^{(-)}$ $=-\left\langle\vec{S}_{b_{j}}\right\rangle(+)$ are self-consistent solutions with all $J_{a_{i} b_{j}} \equiv J_{a_{i} b_{j}}^{(-)} \equiv-J_{a_{i} b_{j}}^{(+)}<0$. This follows because if the $J_{a_{i b j}}$ change sign and if the $\left\{\left\langle\overrightarrow{\mathrm{S}}_{a_{i}}\right\rangle\right\}$ do not change by the definition of $\overrightarrow{\mathrm{H}}_{b_{i}}$ we have $\overrightarrow{\mathrm{H}}_{b_{i}} \rightarrow-\overrightarrow{\mathrm{H}}_{b_{i}}$ which makes $\left\langle\vec{S}_{b_{j}}\right\rangle--\left\langle\vec{S}_{b_{j}}\right\rangle$ by Eqs. (C6) and (C3). This by the definition of $\overrightarrow{\mathrm{H}}_{a_{i}}$ and Eq. (C1) is self-consistent with the original assumption $\left\langle\overrightarrow{\mathrm{S}}_{a_{i}}\right\rangle$ $-\left\langle\vec{S}_{a_{i}}\right\rangle$. A similar argument shows that $\left\langle A_{a_{j}}\right\rangle$ 
and $\left\langle B_{b_{j}}\right\rangle$ are invariant under $J_{a_{i} b_{j}} \rightarrow-J_{a_{i b_{j}}}$, where $A_{a_{i}}$ and $B_{b_{j}}$ are scalar operators. In particular $Z_{a_{i}}$ and $Z_{b_{j}}$ are invariant.

From this we conclude (with $B=0$ ) that $q, f, u, s$, and $c$ are left invariant under $J_{a_{i} b_{j}}--J_{a_{i} b_{j}}$, but neither $\overrightarrow{\mathrm{m}}$ nor $\chi$ would be left unchanged by this change of sign of the $J$ 's.

It should be noted that this proof depends upon the existence of two equivalent sublattices.

\section{APPENDIX D: PARAMAGNETIC AND FERROMAGNETIC CURIE TEMPERATURES IN LMFA}

We first derive the paramagnetic Curie temperature. We assume $\overrightarrow{\mathrm{B}}=B_{z} \hat{z}$ and that the system magnetizes in the $z$ direction and we use

$$
\left\langle S_{j z}\right\rangle=\operatorname{Tr}\left(e^{-\beta h_{j}} S_{j z}\right) / \operatorname{Tr}\left(e^{-\beta h_{j}}\right),
$$

where

$$
h_{j}=-\sum_{i} J_{i j}\left\langle\overrightarrow{\mathrm{S}}_{i}\right\rangle \cdot \overrightarrow{\mathrm{S}}_{j}-D\left(\hat{k}_{j} \cdot \overrightarrow{\mathrm{S}}_{j}\right)^{2}-B_{z} S_{j z} \cdot
$$

Expanding each term to $O\left(\beta^{2}\right)$ and using

$$
\begin{aligned}
& \left\langle S_{j z}\right\rangle=\beta\left\langle S_{j z}\right\rangle_{0}^{\prime}+\frac{1}{2} \beta^{2}\left\langle S_{j z}\right\rangle_{0}^{\prime \prime}\left(\text { since }\left\langle S_{j z}\right\rangle_{\beta=0}=0\right), \\
& \operatorname{Tr} h_{j}=-2 D, \operatorname{Tr} S_{j z}=0, \\
& \operatorname{Tr}\left(h_{j} S_{j z}\right)=-2\left(\sum_{i} J_{i j}\left\langle S_{i z}\right\rangle+B_{z}\right), \\
& \operatorname{Tr}\left(h_{j}^{2} S_{j z}\right)=2 B_{z} D\left(k_{j z}^{2}+1\right) \text { to } O\left(\beta^{0}\right),
\end{aligned}
$$

we have to $O\left(\beta^{2}\right)$,

$$
\begin{aligned}
\left(\beta\left\langle S_{j z}\right\rangle_{0}^{\prime}+\frac{1}{2} \beta^{2}\left\langle S_{j z}\right\rangle_{0}^{\prime \prime}\right)(3+2 \beta D) & \\
& =2 \beta\left(\sum_{i} J_{i j}\left\langle S_{i z}\right\rangle+B_{z}\right)+\beta^{2} B_{k} D\left(k_{j z}^{2}+1\right) .
\end{aligned}
$$

Equating coefficients of $\beta$ and $\beta^{2}$ we have

$$
\begin{aligned}
3 \beta\left\langle S_{j z}\right\rangle_{0}^{\prime}=2 \beta B_{z}, & \\
2 \beta^{2} D\left\langle S_{j z}\right\rangle_{0}^{\prime}+\frac{3}{2} \beta^{2}\left\langle S_{j z}\right\rangle_{0}^{\prime \prime}= & 2 \beta^{2} \sum_{i} J_{i j}\left\langle S_{i z}\right\rangle_{0}^{\prime} \\
& +\beta^{2} B_{z} D\left(k_{j z}^{2}+1\right) .
\end{aligned}
$$

Using Eq. (D4), Eq. (D5) becomes

$$
\frac{1}{2} \beta^{2}\left\langle S_{j z}\right\rangle_{0}^{\prime \prime}=\frac{4}{9} \beta^{2} Z J B_{z}+\frac{1}{3} \beta^{2} B_{z} D\left(k_{j z}^{2}-\frac{1}{3}\right) .
$$

Thus, correct to $O\left(\beta^{2}\right)$ we have

$$
\left\langle S_{j z}\right\rangle=\frac{2}{3} \beta B_{z}\left[1+\frac{2}{3} \beta Z J+\frac{1}{2} \beta D\left(k_{j z}^{2}-\frac{1}{3}\right)\right] .
$$

Because $m_{z}=(1 / N) \sum_{j}\left\langle S_{j z}\right\rangle$ and $(1 / N) \sum_{j} k_{j z}^{2}=\frac{1}{3}$, we have

$$
m_{z}=\frac{2}{3} \beta B_{\varepsilon}\left(1+\frac{2}{3} \beta Z J\right) \text { to } O\left(\beta^{2}\right),
$$

from which we identify the paramagnetic Curie temperature $\theta_{p}$,

$$
\Theta_{p}=\frac{2}{3}(Z J / k) \text { to } O\left(\beta^{2}\right) \text {. }
$$

Note that $\Theta_{p}$ is independent of $D$. We can only be certain of the validity of (D8) when $\beta B_{z}, \beta D$, and $\beta Z J$ are $\ll 1$. Note also that this $D$ independence depends critically on $(1 / N) \sum_{j} k_{j z}^{2}-\frac{1}{3}=0$, i.e., on the random anisotropy model.

To obtain the ferromagnetic Curie temperature, we set $B_{g}=0$ and seek a nonzero but arbitrarily small $m_{z}$. We do this calculation only for $Z j \gg 1$. Explicitly, we expand the traces in Eq. (D1) retaining only linear in $\boldsymbol{D}$ terms. Also, because we are seeking a solution for $\left\langle S_{j s}\right\rangle$ which is nonzero but arbitrarily small, we only retain terms which are linear in $\left\langle S_{j \varepsilon}\right\rangle$. Using the middle three equations (D3) and

$$
\operatorname{Tr}\left(h_{j}^{2} S_{j s}\right)=2 D\left(1+k_{j s}^{2}\right) Z J m_{z}
$$

[to $O(D)$ and retaining terms linear in $\left\langle S_{i z}\right\rangle$ ], we have

$$
\left\langle S_{j z}\right\rangle(3+2 \beta D)=2 \beta \sum_{i} J_{i j}\left\langle S_{i z}\right\rangle+\beta^{2} D m_{z} Z J\left(1+k_{j z}^{2}\right)
$$

to $O(D)$. Then using $m_{z}=(1 / N) \sum_{j}\left\langle S_{j z}\right\rangle, Z J m_{z}$ $=(1 / N) \sum_{i j} J_{i j}\left\langle S_{i k}\right\rangle$, and $(1 / N) \sum_{i} k_{i z}^{2}=\frac{1}{3}$, we have

$$
3 m_{8}\left(1-\frac{2}{3} \beta Z J\right)\left(1+\frac{2}{3} \beta D\right)=0 \text { to } O(D) \text {. }
$$

We thus conclude that the ferromagnetic Curie temperature $T_{C}$ is

$$
T_{C}=\frac{2}{3}(Z J / k) \text { to } O(D) \text {. }
$$

This implies $\left(d T_{C} / d D\right)_{D=0}=0$, so the $T_{C}=T_{C}(D=0)$ $+O\left(D^{2}\right)=\frac{2}{3}(Z J / k)+O\left(D^{2}\right)$, or with $Z=6$,

$$
t_{C}=k T_{c} / D=4 j \text { for } Z j \gg 1 \text {. }
$$

Note by (D9) and (D13) that to $O(D)$ the ferromagnetic Curie temperature equals the paramagnetic Curie temperatures equals the "crystalline" Curie temperature $(D=0)$.

\section{APPENDIX E: INITIATING PROCEDURE}

The set $\left\{\left\langle\vec{S}_{j}\right\rangle\right\}$ used to start the iteration procedure was chosen so that each $\left\langle\mathrm{S}_{j}\right\rangle$ lies along the axis of the random uniaxial anisotropy directions. The purpose of this procedure was to stimulate the system to converge to spin-glass-like states at low temperatures. Specifically, the initial $\left\langle\mathbb{S}_{j}\right\rangle$ were chosen such that

$$
\left\langle\mathbf{S}_{j}\right\rangle=\epsilon_{j} \hat{k}_{j},
$$

where $\epsilon_{j}= \pm 1$. The $\epsilon_{j}$ were chosen so that the $\left\langle\vec{S}_{j}\right\rangle$ were self-consistent zero-temperature solutions for $j \rightarrow 0$ and $\overrightarrow{\mathrm{B}} \rightarrow 0 ; \epsilon_{j}=+1$ if $C_{j}$, the $\hat{k}_{j}$ component of the molecular field (at site $j$ ), is in the positive $\hat{k}_{j}$ direction and $\epsilon_{j}=-1$ if $C_{j}$ is in the negative $\hat{k}_{j}$ direction. Because the $C_{j}$ are given by 


$$
\begin{aligned}
C_{j} & =\left(\sum_{i(n n \text { to } j)} J_{i j}\left\langle\mathrm{~S}_{i}\right\rangle\right) \cdot \hat{k}_{j} \\
& =\sum_{i\left(n n t_{0} j\right)} J_{i j} \epsilon_{i} \hat{k}_{i} \cdot \hat{k}_{j},
\end{aligned}
$$

it follows that

$$
\epsilon_{j}=\frac{\sum_{i(n n \text { to } j)} J_{i j} \epsilon_{i} \hat{k}_{i} \cdot \hat{k}_{j}}{\sum_{i(n n \text { to } j)} J_{i j} \epsilon_{i} \hat{k}_{i} \cdot \hat{k}_{j}} .
$$

Equation (E3) was then iterated, for fixed $\left\{\hat{k}_{i}\right\}$ and $J_{i j}$, to obtain a self-consistent set of $\left\{\epsilon_{i}\right\}$. To start this iteration procedure, an initial set of $\left\{\epsilon_{i}\right\}$ was used in which the $\epsilon_{i}$ were randomly chosen to be \pm 1 .

As stated above, the purpose of this initiating procedure was to stimulate the system to converge to spin-glass-like states at low temperatures; however, this same procedure was used at temperatures well above $T_{C}$ because at those temperatures convergence was rapid and this procedure was as good as any.
*On sabbatical leave from the South Dakota School of Mines and Technology, Rapid City, S. D. 57701. $\dagger$ Present address: Harvard University, Cambridge, Mass. 02138.

${ }^{1}$ S. F. Edwards and P. W. Anderson, J. Phys. F $\underline{5}$, 965 (1975).

${ }^{2}$ R. Harris, M. Plischke, and M. J. Zuckermann, Phys. Rev. Lett. 31, 160 (1973).

${ }^{3}$ G. S. Cargill III, Solid State Phys. 30, 227 (1975).

${ }^{4}$ R. J. Elliott, J. A. Krumhans1, and P. L. Leath, Rev. Mod. Phys. 46, 465 (1974).

${ }^{5}$ M. F. Thorpe and D. Beeman, Phys. Rev. B 14, 188 (1976); R. Brout, Phys. Rev. 115, 824 (1959).

${ }^{6}$ D. Sherrington, AIP Conf. Proc. 29,224 (1975).

${ }^{7}$ For example, see, P. W. Anderson, Amorphous Magnetism II, edited by R. A. Levy and R. Hasegawa (Plenum, New York, 1977), p. 235; G. Heber, Appl. Phys. 10, 101 (1976); H. Müske and G. Heber, J. Phys. F 6 , 71353 (1976); K. H. Fischer, Physica (Utr.) 86-88B, 813 (1977), and references cited therein.

${ }^{8}$ L. E. Wenger and P. H. Keesom, AIP Conf. Proc. $\underline{29}$, 233 (1975).

${ }^{9}$ D. Sherrington and B. W. Southern, J. Phys. F $\underline{5}$, L49 (1975).

${ }^{10}$ K. H. Fischer, Phys. Rev. Lett. 34, 1438 (1975).

${ }^{11}$ A. Madhukar, J. Phys. (Paris) 35, C4 (1974); C. Kittel, Quantum Theory of Solids (Wiley, New York, 1963), p. 360.

${ }^{12}$ K. Binder and K. Schröder, Phys. Rev. B $\underline{14}, 2142$ (1976); Phys. Lett. A 57, 177 (1976).

${ }^{13}$ L. R. Walker and R. E. Walstedt, Phys. Rev. Lett. 38 , 514 (1977).

${ }^{14} \mathrm{~J}$. M. Luttinger, Phys. Rev. Lett. 37, 778 (1976).
${ }^{15}$ R. Harris and D. Zobin, AIP Conf. Proc. 29, 156 (1975); J. Phys. F 7, 337 (1977); R. Harris, B. G. Muliman, and D. Zobin, Physica (Utr.) 86-88B, 755 (1977).

${ }^{16}$ R. W. Cochrane, R. Harris, M. Plischke, D. Zobin, and M. J. Zuckermann, J. Phys. F 5, 763 (1975).

${ }^{17}$ M. C. Chi and R. Alben, AIP Conf. Proc. 34,316 (1976); J. Appl. Phys. 48, 2987 (1977).

${ }^{18}$ R. Harris and S. Sung (private communication).

${ }^{19}$ J. D. Patterson, G. R. Gruzalski, and D. J. Sellmyer (unpublished); see also Bull. Am. Phys. Soc. 22, 3 266 (1977).

${ }^{20} \mathrm{R}$. Harris and S. Sung (private communication) have informed us that recent Monte Carlo simulations give free energies for random ferromagnetic states about $15 \%$ lower than for spin-glass-like states.

${ }^{21}$ E. Callan, Y. J. Liu, and J. R. Cullen, Bull. Am. Phys. Soc. 22, 4572 (1977); and Phys. Rev. B 16, 263 (1977).

${ }^{22}$ M. I. Darby, J. Noncryst. Solids 20, 357 (1976).

${ }^{23}$ P. M. Richards, Phys. Lett. A 55, 121 (1975).

${ }^{24}$ Robert M. White, Quantum Theory of Magnetism (McGraw-Hill, New York, 1970), pp. 44-51 and 103.

${ }^{25} \mathrm{~K}$. Yosida, J. Appl. Phys. 39, 511 (1968).

${ }^{26}$ R. W. Cochrane, R. Harris, and M. Plischke, J. Noncryst. Solids 15, 279 (1974).

${ }^{27}$ Michael W. Klein, Phys. Rev. B 14, 5008 (1976).

${ }^{28}$ A. E. Clark, AIP Conf. Proc. 18, 1015 (1974).

${ }^{29}$ See, e.g., J. J. Rhyne, J. H. Schelleng, and N. C. Koon, Phys. Rev. B 10, 4672 (1974); and G. R. Gruzalski and D. J. Sellmyer (unpublished).

${ }^{30} \mathrm{~J} . \mathrm{M}$ D. Coey and S. von Molnar (unpublished).

${ }^{31} \mathrm{~S}$. V. Tyablikov, Methods in the Quantum Theory of Magnetism (Plenum, New York, 1967), p. 175. 